\title{
Non-fasting triglyceride levels in the Korean population with and without ischemic heart disease and cerebrovascular disease
}

\author{
Seo Young Lee ${ }^{1, *}$, Bo Kyung Koo ${ }^{1,2,}$, , and Min Kyong Moon ${ }^{1,2}$
}

${ }^{1}$ Department of Internal Medicine, Seoul National University College of Medicine, Seoul; ${ }^{2}$ Department of Internal Medicine, Seoul Metropolitan Government Seoul National University Boramae Medical Center, Seoul, Korea

Received: September 5, 2016 Revised : January 25, 2017 Accepted: February 19, 2017

\section{Correspondence to}

\section{Min Kyong Moon, M.D.}

Department of Internal Medicine, Seoul Metropolitan Government Seoul National University Boramae Medical Center, 20 Boramae-ro 5-gil, Dongjak-gu, Seoul 07061, Korea

Tel: +82-2-870-2226

Fax: +82-2-831-2826

E-mail:mkmoon@snu.ac.kr

*These authors contributed equally to this work.
Background/Aims: Associations between non-fasting triglyceride (TG) levels and a risk of ischemic heart disease (IHD) and cerebrovascular accident (CVA) have been suggested in Caucasians. We aimed to investigate whether non-fasting TG levels reflect the risk of IHD/CVA in Koreans.

Methods: We conducted an analysis of patients aged $\geq 30$ years from the nationwide survey database. Fasting TG was defined as a measurement taken $\geq 12$ hours since the last meal. Non-fasting TG was categorized by fasting duration of 0 to 3 , 4 to 7 , and 8 to 11 hours.

Results: In subjects without history of IHD/CVA, diabetes, or lipid-lowering medication, the TG level was significantly elevated for 7 hours in men compared to fasting TG levels $(p=0.011)$; the mean TG levels were $154.9 \mathrm{mg} / \mathrm{dL}$ (standard error [SE], 13.0), 177.0 mg/dL (SE, 12.1), $148.8 \mathrm{mg} / \mathrm{dL}$ (SE, 2.8), and 141.5 mg/dL (SE, 1.4) for 0 to 3,4 to 7,8 to 11 , and $\geq 12$ hours' fasting, respectively. In women, there was no difference in TG level according to fasting duration after adjustment for confounders. In men without diabetes, the TG level from 4 to 7 hours' fasting showed a significant difference between subjects with or without IHD/CVA even after adjustments for age, body mass index, lipid medication, exercise, and dietary factors (215.1 mg/dL vs. $177.3 \mathrm{mg} / \mathrm{dL}, p<0.001$ ).

Conclusions: In men, non-fasting TG levels from 4 to 7 hours' fasting were significantly associated with IHD/CVA, and were superior to fasting TG levels level in the significant association with the history of IHD or CVA.

Keywords: Non-fasting triglyceride; Cardiovascular disease; Myocardial ischemia; Fasting duration; Fasting triglyceride

\section{INTRODUCTION}

Triglyceride (TG) level was reported to be associated with coronary artery disease, even after adjustment for established risk factors including low density lipoprotein cholesterol (LDL-C) in several prospective cohort studies [1-3]. Furthermore, TG-lowering treatment caused a reduction in cardiovascular disease (CVD) risk in a selected population who had hypertriglyceridemia with or without a low high density lipoprotein cholesterol (HDL-C) level $[4,5]$.

Recently, the non-fasting TG level has been reported to be independently associated with the risk of a cardiovascular event from $>10$ years of prospective cohort studies in Caucasian populations [6,7]. In addition, non-fasting TG was superior to fasting TG level for 
predicting the risk of CVD [6]. In fact, most individuals are in the non-fasting state except for a few hours in the morning [8]; thus, non-fasting TG level might reflect the usual exposure level of TG in our bodies, and be more closely associated with the risk of atherosclerosis. TG levels normally return to baseline fasting levels 10 hours after a fat-containing meal [8]; however, in the general population with a normal diet, TG levels returned to baseline 6 hours after the last meal in the Caucasian population [7].

TG level can differ between races. Lipoprotein lipase activity and postprandial TG level was reported to be differ according to ethnicity [9]. However, there are very limited data on postprandial TG in Asian populations. Thus, we aimed to analyze the change in lipid level including TG level according fasting duration in the general Korean population using a nationwide survey. We also investigated whether the non-fasting TG level reflects the risk of ischemic heart disease (IHD) and cerebrovascular accident (CVA).

\section{METHODS}

\section{Study participants}

The Korean National Health and Nutrition Examination Survey (KNHANES), a nationwide cross-sectional survey conducted by the Ministry of Health and Welfare of Korea from January 2007 to December 2013, was used. In KNHANES, the sampling design was used based on household registries stratified by geographical area, sex, and age groups to be representative of the Korean population.

Among 38,503 individuals aged $\geq 30$ years, we excluded subjects with (1) a TG level > 1,000 mg/dL to rule out cases with hereditary hypertriglyceridemia [10], (2) a history of heavy alcohol consumption, (3) presence of thyroid disease, (4) estimating glomerular filtration rate < 30 $\mathrm{mL} / \mathrm{min} / 1.73 \mathrm{~m}^{2}$ or (5) no response to the questionnaire on IHD/CVA history. A final total of 28,094 subjects aged $\geq 30$ years were included in the analysis. Fasting duration of each subject was evaluated using the self-questionnaire.

The study was approved by the Institutional Review Board (IRB) of Korea Centers for Disease Control and Prevention annually and the IRB approval numbers were 2007-O2CON-O4-P, 2008-04EXP-01-C, 2009-01CON-03-
2C, 2010-02CON-21-C, 2011-02CON-o6-C, 2012-o1EXPO1-2C, and 2013-07CON-03-4C.

Informed consents were waived due to the retrospective nature of this study.

\section{Measurement of metabolic parameters and disease definitions}

Blood samples were drawn from the antecubital vein, processed, immediately refrigerated at $2^{\circ} \mathrm{C}$ to $8^{\circ} \mathrm{C}$, and sent to a central laboratory. Total cholesterol, HDL-C, LDL-C, and TG levels were measured enzymatically using serum (Hitachi Automatic Analyzer 7600, Hitachi, Tokyo, Japan). Glycated hemoglobin was measured using high performance liquid chromatography (HLC${ }_{723} \mathrm{G}_{7}$, Tosoh, Tokyo, Japan). Fasting TG was defined as the TG level measured $\geq 12$ hours since the last meal. Non-fasting TG was categorized by a fasting duration of o to 3, 4 to 7, or 8 to 11 hours. Blood pressure was measured using a mercury sphygmomanometer (Baumanometer, Baum, Copiague, NY, USA) to the nearest 2 mmHg. Hypertension was defined when systolic blood pressure was $\geq 140 \mathrm{mmHg}$, diastolic blood pressure was $\geq 90 \mathrm{mmHg}$ or the patient was receiving anti-hypertensive medicine. Diabetes was defined in subjects with an 8-hour fasting plasma glucose level of $\geq 126 \mathrm{mg} / \mathrm{dL}$, a random blood glucose level $\geq 200 \mathrm{mg} / \mathrm{dL}, \mathrm{HbAlc} \geq 6.5 \%$ or taking antidiabetic drugs, including insulin [11]. CVA was determined when a subject was ever told that they had a stroke by a healthcare professional, and IHD was determined when a subject was ever told that they had coronary heart disease or angina by a healthcare professional. Smoking and alcohol-drinking history, and menopausal status were evaluated using the health interview survey, and heavy drinkers were defined as those who consumed over $30 \mathrm{~g} /$ day alcohol in men and over 20 $\mathrm{g} /$ day in women [12,13].

Nutritional information was collected using 24-hour recall administered by a trained dietary interviewer in the Korea Health Industry Development Institute. The 24-hour recall was based on a 1-day diary of daily food intake and nutrients were quantified using the Database Management System (Korea Health Industry Development Institute, Seoul, Korea); and daily fat and carbohydrate intake in each individual was analyzed after adjustment for body weight (g/kg/day). Those who stated performing 30-minute moderate-intensity exercise $\geq 5$ 
days a week, 20-minute high-intensity exercise $\geq 3$ days a week, or 30 -minute walking $\geq 5$ days a week were considered to have attained the exercise goal.

\section{Statistical analyses}

Sampling weights were used to account for the complex sampling, and all analyses were conducted independently according to sex. Statistical analyses were conducted using SPSS version 18.0 software for Windows (SPSS Inc., Chicago, IL, USA). The results are presented as means or as prevalence with standard error (SE). Binary logistic regression and linear regression analysis for categorical and continuous variables, respectively, were used to compare the TG level according to the fasting duration in each subgroup. Complex sample regression analysis applying weights with adjustment for age, body mass index (BMI), lipid-lowering medication, exercise, and dietary factors was performed to compare the TG level according to the IHD or CVA (IHD/CVA) history. Statistical significance was defined as $p<0.05$.

\section{RESULTS}

\section{Baseline characteristics of the study population}

Among the entire study population (unweighted $n=$
28,094), 5.1\% had IHD/CVA history. Subjects with IHD/ CVA were significantly older compared to those without (64.6 years [SE, 0.4] vs. 49.9 years [SE, 0.1], $p<0.001$ ). Established coronary risk factors such as hypertension, diabetes mellitus, and lipid-lowering medications were more prevalent in subjects with IHD/CVA (Table 1). They also had a significantly higher serum fasting TG level (145.9 mg/dL [SE, 3.1] vs. 130.1 mg/dL [SE, 0.7], $p<$ 0.001 ) and a significantly lower HDL-C level (44.1 mg/dL [SE, 0.5] vs. $46.6 \mathrm{mg} / \mathrm{dL}$ [SE, o.2] in men; $48.7 \mathrm{mg} / \mathrm{dL}$ [SE, $0.6]$ vs. $53.4 \mathrm{mg} / \mathrm{dL}$ [SE, o.1] in women; $p<0.001$ in both sex) compared to subjects without IHD/CVA. Among women, the prevalence of menopause was $58.2 \%$ (SE, $2.2 \%$ ) and $30.2 \%$ (SE, $0.5 \%$ ) in subjects with and without IHD/CVA, respectively $(p<0.001)$.

\section{TG levels according to fasting time}

At first, we investigated TG levels according to fasting duration in the general population. For this analysis, we excluded the subjects with IHD/CVA for generalization to the Korean population. Considering that the limited number of participants with a fasting duration of $<12$ hours might weaken the statistical significance (Fig. 1), we categorized fasting duration into o to 3,4 to 7,8 to 11 hours, and $\geq 12$ hours, and compared TG levels from o to 3,4 to 7 , and 8 to 11 hours with those from $\geq 12$ hours'
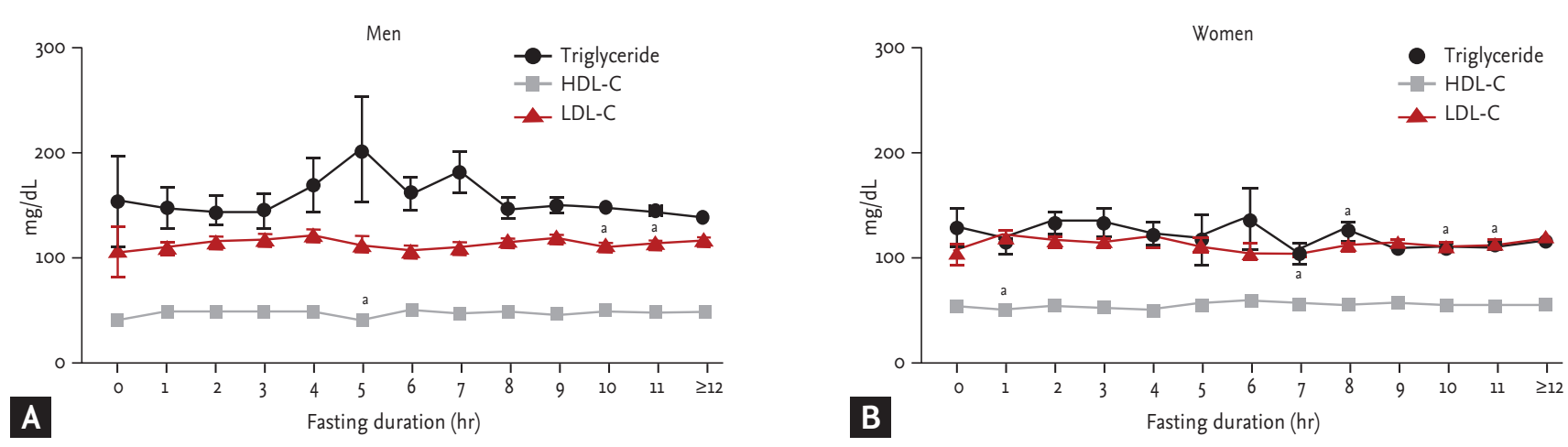

\begin{tabular}{|c|c|c|c|c|c|c|c|c|c|c|c|c|c|c|}
\hline No. of & ec & & & & & & & & & & & & & \\
\hline & o hr & $1 \mathrm{hr}$ & $2 \mathrm{hr}$ & $3 \mathrm{hr}$ & $4 \mathrm{hr}$ & $5 \mathrm{hr}$ & $6 \mathrm{hr}$ & $7 \mathrm{hr}$ & $8 \mathrm{hr}$ & $9 \mathrm{hr}$ & $10 \mathrm{hr}$ & $11 \mathrm{hr}$ & $\geq 12 \mathrm{hr}$ & Total \\
\hline Men & 4 & 34 & 34 & 30 & 31 & 19 & 23 & 48 & 105 & 234 & 506 & 807 & 6,120 & 7,995 \\
\hline Women & 11 & 59 & 87 & 51 & 35 & 11 & 16 & 46 & 105 & 245 & 664 & 1,305 & 12,148 & 14,783 \\
\hline
\end{tabular}

Figure 1. Lipid level in subjects without ischemic heart disease/cerebrovascular accident, diabetes, or lipid-lowering agent according to fasting duration. (A) In men, TG level from every fasting duration was not statistically different from TG level obtained from $\geq 12$ hours' fasting. (B) In women, TG level from 8 hours' fasting was significantly higher than that from $\geq$ 12 hours' fasting (age-adjusted $p=0.017$ ). LDL-C from 10 and 11 hours' fasting was significantly lower than LDL-C from $\geq 12$ hours' fasting in both genders (age-adjusted $p<0.05$ ). HDL-C, high density lipoprotein cholesterol; LDL-C, low density lipo-

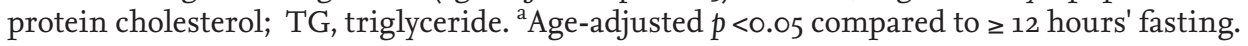


Table 1. Baseline characteristics of study population

\begin{tabular}{|c|c|c|c|}
\hline Clinical parameter & Control & IHD/CVA & $p$ value \\
\hline Unweighted number & 26,672 & 1,422 & - \\
\hline Age, yr & $49.9 \pm 0.1$ & $64.6 \pm 0.4$ & $<0.001$ \\
\hline Male sex, \% & $41.2 \pm 0.3$ & $51.5 \pm 1.6$ & $<0.001$ \\
\hline Body mass index, $\mathrm{kg} / \mathrm{m}^{2}$ & $23.78 \pm 0.03$ & $24.55 \pm 0.09$ & $<0.001$ \\
\hline \multicolumn{4}{|l|}{ Waist circumference, $\mathrm{cm}$} \\
\hline Male & $84.3 \pm 0.1$ & $86.4 \pm 0.4$ & $<0.001$ \\
\hline Female & $79.1 \pm 0.1$ & $84.7 \pm 0.4$ & $<0.001$ \\
\hline Systolic blood pressure, $\mathrm{mmHg}$ & $117.8 \pm 0.2$ & $127.0 \pm 0.6$ & $<0.001$ \\
\hline Diastolic blood pressure, mmHg & $76.3 \pm 0.1$ & $76.5 \pm 0.4$ & 0.509 \\
\hline Fasting glucose, mg/dL & $98.0 \pm 0.2$ & $107.1 \pm 0.9$ & $<0.001$ \\
\hline Total cholesterol, mg/dL & $191.2 \pm 0.3$ & $178.9 \pm 1.4$ & $<0.001$ \\
\hline Fasting triglycerides, mg/dL & $130.1 \pm 0.7$ & $145 \cdot 9 \pm 3.1$ & $<0.001$ \\
\hline \multicolumn{4}{|l|}{ HDL-C, mg/dL } \\
\hline Male & $46.6 \pm 0.2$ & $44.1 \pm 0.5$ & $<0.001$ \\
\hline Female & $53 \cdot 4 \pm 0.1$ & $48.7 \pm 0.6$ & $<0.001$ \\
\hline $\mathrm{LDL}-\mathrm{C}, \mathrm{mg} / \mathrm{dL}$ & $115.1 \pm 0.3$ & $104 \cdot 0 \pm 1.3$ & $<0.001$ \\
\hline Serum creatinine, mg/dL & $0.84 \pm 0.00$ & $0.94 \pm 0.01$ & $<0.001$ \\
\hline Aspartate aminotransferase, IU/L & $21.9 \pm 0.1$ & $24.1 \pm 0.4$ & $<0.001$ \\
\hline Alanine aminotransferase, IU/L & $21.8 \pm 0.1$ & $22.9 \pm 0.5$ & $<0.001$ \\
\hline Hypertension, \% & $27.7 \pm 0.4$ & $64.3 \pm 1.6$ & $<0.001$ \\
\hline Diabetes mellitus, \% & $10.1 \pm 0.2$ & $29.9 \pm 1.5$ & $<0.001$ \\
\hline Lipid medication, \% & $4 \cdot 3 \pm 0.1$ & $23 \cdot 3 \pm 1.4$ & $<0.001$ \\
\hline Current cigarette smoker, \% & $27 \cdot 3 \pm 0.4$ & $28.8 \pm 1.6$ & 0.330 \\
\hline Regular exercise, $\%^{\mathrm{a}}$ & $48.1 \pm 0.4$ & $48.7 \pm 1.7$ & 0.725 \\
\hline \multicolumn{4}{|l|}{ Menopausal status (female) } \\
\hline Menopause, \% & $30.2 \pm 0.5$ & $58.2 \pm 2.2$ & $<0.001$ \\
\hline Menopause + HRT, \% & $8.0 \pm 0.2$ & $19.7 \pm 1.7$ & $<0.001$ \\
\hline
\end{tabular}

Values are presented as mean $\pm \mathrm{SE}$.

IHD, ischemic heart disease; CVA, cerebrovascular accident; HDL-C, high density lipoprotein cholesterol; LDL-C, low density lipoprotein cholesterol; HRT, hormone replacement therapy.

${ }^{2}$ Those who stated performing 30-minute moderate-intensity exercise $\geq 5$ days a week, 20-minute high-intensity exercise $\geq 3$ days a week or 30-minute walking $\geq 5$ days a week were considered to have attained the aerobic exercise goal.

fasting. As age and BMI independently affected TG level ( $p<0.001$ in both), we compared the TG level according to fasting duration with adjustment for age and BMI. In men, mean TG levels were $156.0 \mathrm{mg} / \mathrm{dL}$ (SE, 10.7), 181.9 $\mathrm{mg} / \mathrm{dL}$ (SE, 11.6), $152.9 \mathrm{mg} / \mathrm{dL}$ (SE, 2.7), and $146.8 \mathrm{mg} / \mathrm{dL}$ (SE, 1.4) for 0 to 3,4 to 7,8 to 11 , and $\geq 12$ hours fasting, respectively. TG levels from 4 to 7 and 8 to 11 hours' fasting were significantly higher compared to those of $\geq$ 12 hours fasting (age and BMI adjusted $p=0.027$ and $p$ $=0.003$, respectively), whose statistical significance was maintained even after additional adjustment for ex- ercise, and daily carbohydrate and fat intake per body weight $(p=0.033$ and $p=0.004$ ) (Table 2 ).

As diabetes [14] and lipid-lowering medication [15] are strong confounders of TG level, we subsequently analyzed the TG levels according to fasting duration only with those who had no diabetes and did not take lipid-lowering medication. TG levels from 4 to 7 and 8 to 11 hours' fasting were higher than those of $\geq 12$ hours fasting with statistical significance $(p=0.001$ and $p=0.004$, respectively). However, after adjustment for age, BMI, exercise, and daily carbohydrate and fat intake, only TG 


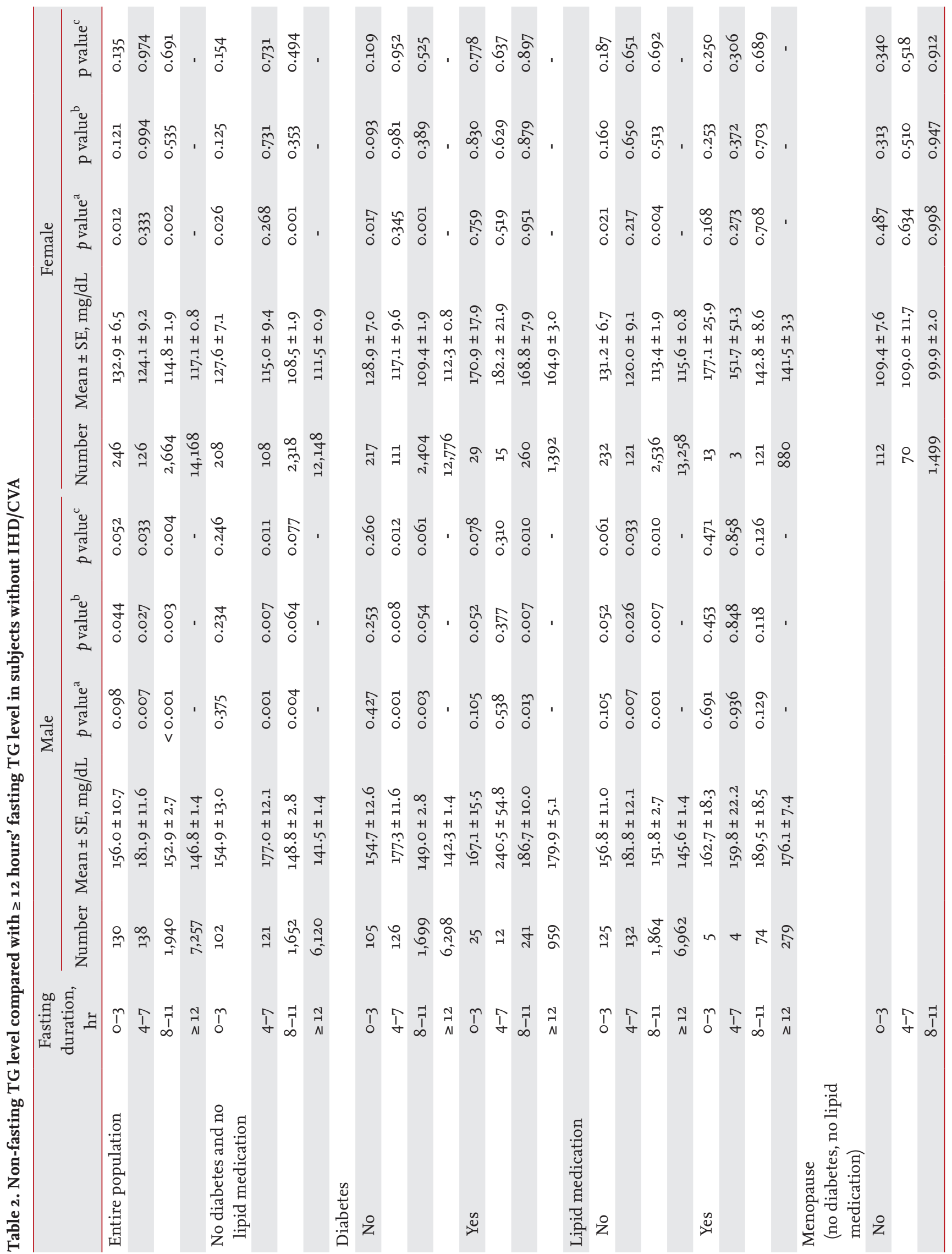




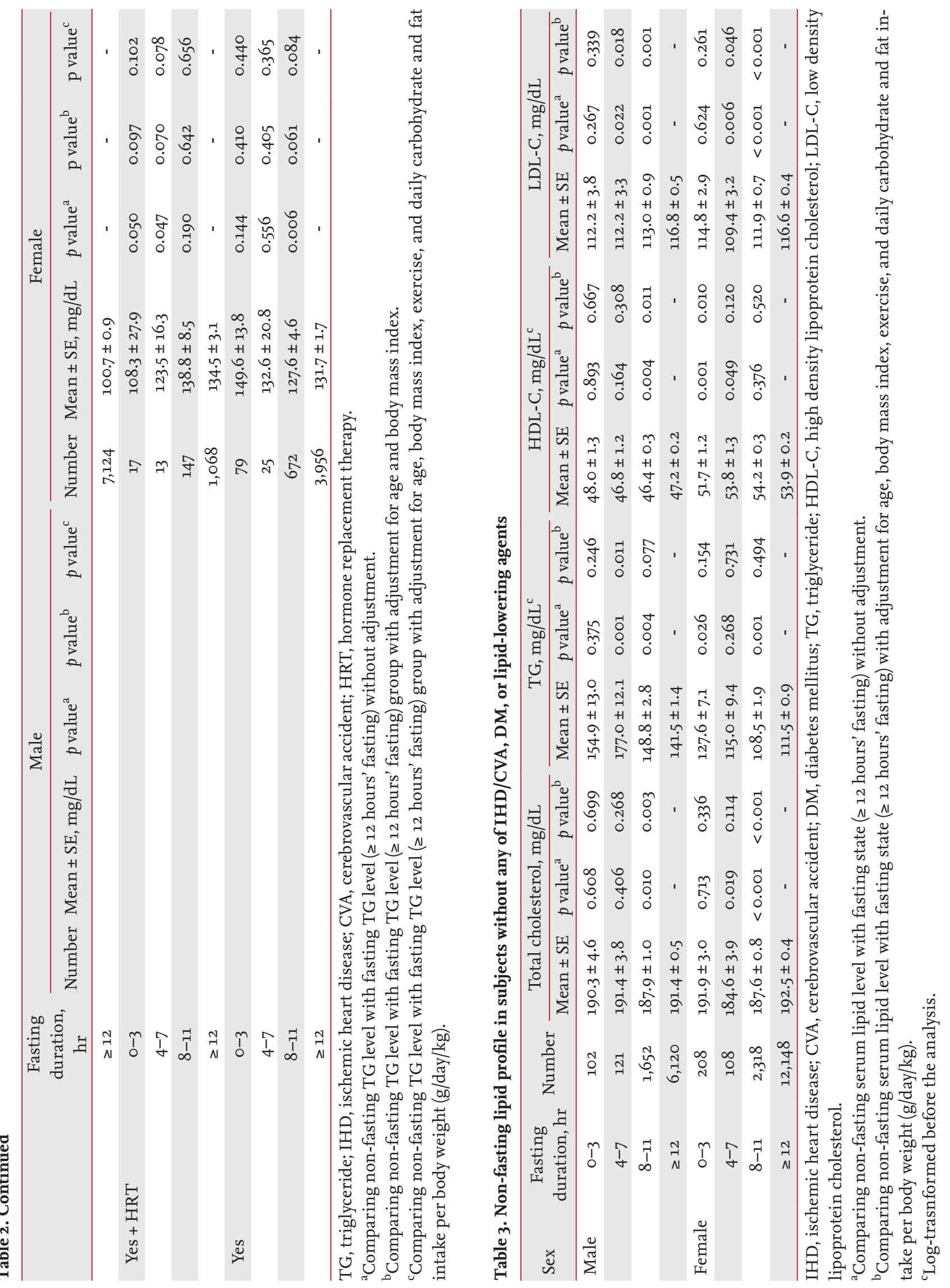




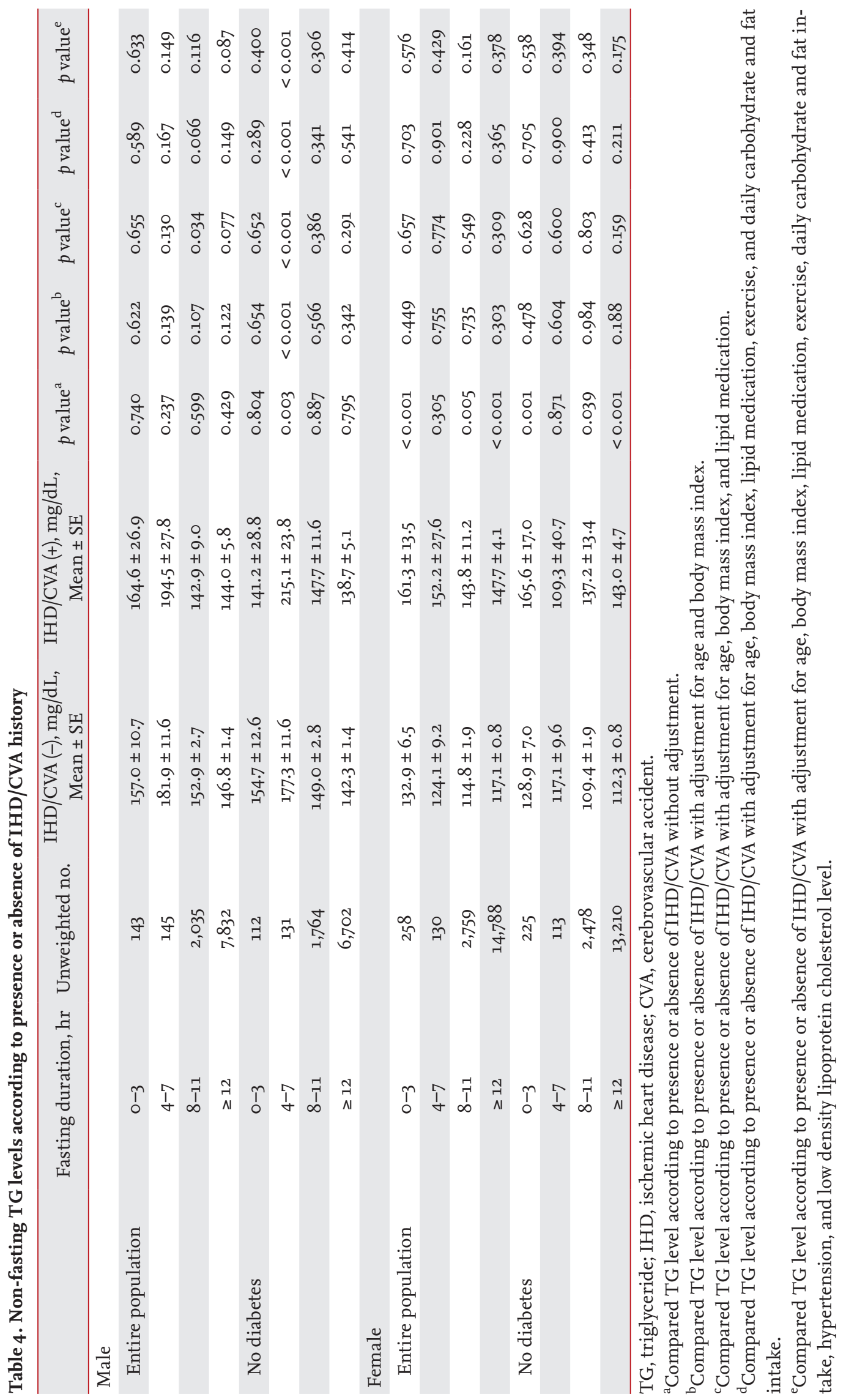


levels from 4 to 7 hours' fasting were significantly higher compared to fasting TG levels $(p=0.011$ ) (Table 2). Men with diabetes also showed tendency similar trend; however, there was no statistically significant difference between TG levels from 4 to 7 hours' fasting and fasting TG levels due to the limited number of study subjects with diabetes (Table 2). In them, TG levels from 8 to 11 hours' fasting was significantly higher than fasting TG levels even after adjustment for age, BMI, exercise, and daily carbohydrate and fat intake $(p=0.010)$ (Table 2).

In women, mean TG levels were $132.9 \mathrm{mg} / \mathrm{dL}$ (SE, 6.5), $124.1 \mathrm{mg} / \mathrm{dL}$ (SE, 9.2), $114.8 \mathrm{mg} / \mathrm{dL}$ (SE, 1.9), and $117.1 \mathrm{mg} /$ $\mathrm{dL}$ (SE, o.8) for o to 3, 4 to 7,8 to 11 , and $\geq 12$ hours' fasting, respectively. After adjustment for age and BMI, there was no significant difference in TG levels according to fasting duration (Table 2). Analysis of only non-diabetic women without any lipid-lowering medication or the stratified analysis according to menopausal status also revealed no difference in TG levels according to fasting duration (Table 2).

For analysis of the non-fasting lipid profile other than TG, we only used the study subjects without any history of IHD/CVA, diabetes, or lipid-lowering medication. The HDL-C level from 8 to 11 hours' fasting in men and o to 3 hours' fasting in women was significantly lower compared to that of $\geq 12$ hours' fasting even after adjustment for age, BMI, exercise, and daily carbohydrate and fat intake per body weight $(p=0.011$ and $p=0.010$, respectively) (Table 3). In the case of LDL-C, the level from 4 to 7 hours and 8 to 11 hours' fasting was statistically lower compared to that of $\geq 12$ hours fasting, even after adjusting for covariates in both sexes (Table 3).

\section{Association of non-fasting TG levels with IHD/CVA}

Next, we investigated whether there was a difference in non-fasting TG level according to the presence or absence of IHD/CVA history. In entire men, there was no difference in TG level between subjects with and without IHD/CVA in each fasting duration (Table 4). However, in the case of men without diabetes, TG levels from 4 to 7 hours' fasting was significantly higher in subjects with IHD/CVA (215.1 mg/dL [SE, 23.8]) compared to levels in those without IHD/CVA (177.3 mg/dL [SE, 11.6], $p=$ ○.003). Even after adjusting for age, BMI, lipid medication, exercise, daily carbohydrate and fat intake, hypertension, and LDL-C level, its statistical significance was maintained $(p<0.001)$ (Table 4). By contrast, TG level of $\geq 12$ hours' fasting was $138.7 \mathrm{mg} / \mathrm{dL}$ (SE, 5.1) and $142.3 \mathrm{mg} /$ $\mathrm{dL}$ (SE, 1.4) in non-diabetic men with and without IHD/ CVA, respectively; there was no statistically significant difference between them (Table 4).

In the case of women, those with IHD/CVA had significantly higher TG levels compared to those without IHD/CVA in every fasting duration except 4 to 7 hours, irrespective of diabetes status (Table 4). The statistical difference in TG level according to IHD/CVA did not persist after adjustment for age and lipid-lowering medication (Table 4).

\section{DISCUSSION}

The current study using a nationwide survey representing the Korean population revealed that non-fasting TG level was associated with the previous history of IHD/CVA. Especially in men, the association between non-fasting TG from 4 to 7 hours' fasting and IHD/CVA was maintained even after adjustment for confounders. Furthermore, in this group, non-fasting TG was superior to fasting TG level in the significant association with the previous history of IHD or CVA. By contrast, in the case of women, non-fasting TG or fasting TG level was not associated with IHD/CVA after adjustment for age and BMI.

Considering the usual diet pattern of the general population, most of time we are in a non-fasting state except for the time before breakfast [16]. Therefore, non-fasting TG rather than fasting TG levels might reflect the actual exposure of TG level, which could be associated with the occurrence of the diseases. As the non-fasting TG level is determined by postprandial remnant lipoprotein, which can cause atherosclerosis [17,18], non-fasting TG could be related to the risk of IHD or CVA. Furthermore, high ratio of TG to low density lipoprotein increased susceptibility to oxidation [19] and was associated with increasing factor VII and plasminogen activator inhibitor levels [20], which might promote atherosclerosis and thrombosis.

In terms of TG level according to fasting duration, TG level normally return to baseline fasting levels at 12 hours in men and 4 hours in women after the last meal in our study. Women are known to have lower postpran- 
dial TG than men because of the higher clearance capacity caused by increased lipoprotein lipase activity [21]. The metabolic clearance rate of fatty acids was higher in women compared to men [22]. Even after considering the sex difference in TG level, men in our study showed a relatively longer duration of postprandial TG elevation compared to the Caucasian population [7]. We previously reported that fasting TG is also significantly higher in the Korean population compared to the U.S. population [23]. The reason for Korean men having a higher TG level and a relative longer duration of postprandial TG level compared to the Caucasian population is not known. Genetic polymorphisms in the APOA5 [24] and $A_{3 O C_{3}}[25]$ genes might explain the observed ethnic differences in TG levels, i.e., the $\mathrm{APOA}_{5}-1131 \mathrm{C}$ allele is more prevalent in Asian populations compared to Caucasian populations [24] and the presence of -1131C allele was associated with a high TG level [24]. Different dietary compositions between Asians and Caucasians might also partly explain the difference. Asian populations, including the Korean population, eat more carbohydrate compared to Caucasians [26] and also consume a relative lower proportion of protein [27], which is known to exacerbate postprandial chylomicron levels $[28,29]$. However, considering the sex difference in the duration of postprandial hypertriglyceridemia in our study, other factors aside from genetic factors or carbohydrate consumption rate might be existed. Further analysis is needed to confirm the difference between dietary composition and postprandial lipid levels in different ethnic groups.

In our study, the association between non-fasting TG and IHD/CVA was also different between both sexes. The significant association between non-fasting TG and IHD/CVA in a multivariate model was observed only in men, which was consistent with a previous report from Japan [30]. However, there has been conflicting data on the sex difference in the association between TG level and coronary heart disease in Asia [31] based on meta-analysis in population-based, prospective studies [32]. Considering that this method of crude analysis without adjustment showed that those with IHD/CVA had significantly higher non-fasting TG than women without IHD/CVA, and that there were a very limited number of subjects with fasting $\leq 12$ hours in our study, the association between non-fasting TG level and IHD/CVA should be interpreted with caution.
The association between non-fasting TG and IHD/ CVA was prominent in non-diabetic subjects in our study. However, because there was a relative small number of study subjects with diabetes, it should be interpreted with caution. The insignificant result in diabetic subgroups might be partially explained by secondary hypertriglyceridemia as a consequence of diabetic dyslipidemia [33-36], which can reduce the variance in TG level according to IHD/CVA status.

In the case of LDL-C, its level significantly decreased following 4 to 7 and 8 to 11 hours' fasting after adjusting variables, which was consistent with results from a Copenhagen study group [14]. The decrease in postprandial LDL-C can be explained by transient hemodilution after fluid intake with a meal [14]. This hemodilution effect can be adjusted by the albumin level, however, the lack of information on albumin level in this survey prevented us from correcting the hemodilution effect in LDL-C. The same effect was observed for HDL-C in men, showing a significant decrease level in 8 to 11 hours' fasting, while women showed a decreased level in o to 3 hours' fasting, which was a similar pattern to that of TG. In addition, considering the transient hemodilution after a meal, postprandial TG level might be underestimated in our study.

There are several limitations to this study. First, since this is a cross-sectional study, a causal relationship could not be proven. Second, the questionnaires were self-reported, and there could have recall bias. Patients with relatively mild disease such as angina or transient ischemic attack could have been unintentionally omitted. Another limitation was that the cholesterol levels were not measured repeatedly in each participant according the fasting time. Furthermore, a small number of unweighted subjects measuring TG level with a short postprandial duration weakens the statistical power of our results. Lastly, even though we considered the confounders causing secondary hypertriglyceridemia such as alcohol drinking, thyroid disease, diabetes, and chronic kidney disease, we could not exclude other possible contributors of hypertriglyceridemia such as nephrotic syndrome or medications. Furthermore, we investigated the association between TG level and IHD/ CVA with adjustment for lipid-lowering medication; however, we could not differentiate the fibrate and statin. However, we used the data from a nationwide sur- 
vey using a large number individuals representative of the Korean population. Although we could not show a causal relationship between non-fasting TG level and IHD/CVA risk, we replicated the significant association between non-fasting TG level and CVD risk confirmed in the previous prospective cohorts performed in the Caucasian populations $[6,7]$. To exclude the important confounders of hypertriglyceridemia, we adopted the excluding criteria for heavy alcohol drinking, thyroid disease, and uremia. Furthermore, to investigate the TG levels according to fasting duration, we only included the subjects without any of diabetes or lipid-lowering medication for the analysis.

In conclusion, non-fasting TG level was significantly higher for 7 postprandial hours compared to fasting TG level in men. In men, non-fasting TG levels from 4 to 7 hours' fasting were significantly associated with IHD/ CVA, and it was superior to fasting TG level in the significant association with the previous history of IHD or CVA. By contrast, in the case of women, non-fasting TG or fasting TG was not associated with IHD/CVA after adjustment for age and BMI. These data may be useful to define the appropriate timing of sampling for lipid measurements in individuals in Asia. Furthermore, our study suggests that sampling non-fasting TG might be valuable for detecting an association with atherosclerotic disease in specific subgroups.

\section{KEY MESSAGE}

1. Non-fasting triglyceride (TG) level was significantly higher compared to fasting TG level for 7 postprandial hours in men.

2. In men, non-fasting TG levels from 4 to 7 hours' fasting were significantly associated with ischemic heart disease/cerebrovascular accident (IHD/CVA), and it was superior to fasting TG level in the significant association with the previous history of IHD or CVA.

3. Sampling non-fasting TG might be valuable for detecting an association with atherosclerotic disease in specific subgroups.

\section{Conflict of interest}

No potential conflict of interest relevant to this article was reported.

\section{REFERENCES}

1. Sarwar N, Danesh J, Eiriksdottir G, et al. Triglycerides and the risk of coronary heart disease: 10,158 incident cases among 262,525 participants in 29 Western prospective studies. Circulation 2007;115:450-458.

2. Tirosh A, Rudich A, Shochat T, et al. Changes in triglyceride levels and risk for coronary heart disease in young men. Ann Intern Med 2007;147:377-385.

3. Johansson S, Wilhelmsen L, Lappas G, Rosengren A. High lipid levels and coronary disease in women in Goteborg: outcome and secular trends: a prospective 19 year follow-up in the BEDA*study. Eur Heart J 2003;24:704-716.

4. ACCORD Study Group, Ginsberg HN, Elam MB, et al. Effects of combination lipid therapy in type 2 diabetes mellitus. N Engl J Med 2010;362:1563-1574.

5. Scott R, O'Brien R, Fulcher G, et al. Effects of fenofibrate treatment on cardiovascular disease risk in 9,795 individuals with type 2 diabetes and various components of the metabolic syndrome: the Fenofibrate Intervention and Event Lowering in Diabetes (FIELD) study. Diabetes Care 2009;32:493-498.

6. Bansal S, Buring JE, Rifai N, Mora S, Sacks FM, Ridker PM. Fasting compared with nonfasting triglycerides and risk of cardiovascular events in women. JAMA 2007;298:309-316.

7. Langsted A, Freiberg JJ, Nordestgaard BG. Fasting and nonfasting lipid levels: influence of normal food intake on lipids, lipoproteins, apolipoproteins, and cardiovascular risk prediction. Circulation 2008;118:2047-2056.

8. Cohn JS, McNamara JR, Cohn SD, Ordovas JM, Schaefer EJ. Plasma apolipoprotein changes in the triglyceride-rich lipoprotein fraction of human subjects fed a fatrich meal. J Lipid Res 1988;29:925-936.

9. Bower JF, Deshaies Y, Pfeifer M, Tanenberg RJ, Barakat HA. Ethnic differences in postprandial triglyceride response to a fatty meal and lipoprotein lipase in lean and obese African American and Caucasian women. Metabolism 2002;51:211-217.

10. Jacobson TA, Miller M, Schaefer EJ. Hypertriglyceridemia and cardiovascular risk reduction. Clin Ther 2007;29:763777 .

11. American Diabetes Association. 2. Classification and diagnosis of diabetes. Diabetes Care 2015;38 Suppl:S8-S16.

12. Chalasani N, Younossi Z, Lavine JE, et al. The diagnosis and management of non-alcoholic fatty liver disease: 
practice guideline by the American Gastroenterological Association, American Association for the study of Liver Diseases, and American College of Gastroenterology. Gastroenterology 2012;142:1592-1609.

13. Van de Wiel A. The effect of alcohol on postprandial and fasting triglycerides. Int J Vasc Med 2012;2012:862504.

14. Langsted A, Nordestgaard BG. Nonfasting lipids, lipoproteins, and apolipoproteins in individuals with and without diabetes: 58434 individuals from the Copenhagen General Population study. Clin Chem 2011;57:482-489.

15. Karlson BW, Palmer MK, Nicholls SJ, Lundman P, Barter PJ. A VOYAGER meta-analysis of the impact of statin therapy on low-density lipoprotein cholesterol and triglyceride levels in patients with hypertriglyceridemia. Am J Cardiol 2016;117:1444-1448.

16. Nordestgaard BG, Langsted A, Mora S, et al. Fasting is not routinely required for determination of a lipid profile: clinical and laboratory implications including flagging at desirable concentration cut-points-a joint consensus statement from the European Atherosclerosis Society and European Federation of Clinical Chemistry and Laboratory Medicine. Eur Heart J 2016;37:1944-1958.

17. Zilversmit DB. Atherogenesis: a postprandial phenomenon. Circulation 1979;60:473-485.

18. Varbo A, Benn M, Tybjaerg-Hansen A, Jorgensen AB, Frikke-Schmidt R, Nordestgaard BG. Remnant cholesterol as a causal risk factor for ischemic heart disease. J Am Coll Cardiol 2013;61:427-436.

19. de Graaf J, Hak-Lemmers HL, Hectors MP, Demacker PN, Hendriks JC, Stalenhoef AF. Enhanced susceptibility to in vitro oxidation of the dense low density lipoprotein subfraction in healthy subjects. Arterioscler Thromb 1991;11:298-306.

20. Hypertriglyceridaemia and vascular risk: report of a meeting of physicians and scientists, University College London Medical School. Lancet 1993;342:781-787.

21. Cohn JS, McNamara JR, Cohn SD, Ordovas JM, Schaefer EJ. Postprandial plasma lipoprotein changes in human subjects of different ages. J Lipid Res 1988;29:469-479.

22. Sarac I, Backhouse K, Shojaee-Moradie F, et al. Gender differences in VLDL1 and VLDL2 triglyceride kinetics and fatty acid kinetics in obese postmenopausal women and obese men. J Clin Endocrinol Metab 2012;97:24752481.

23. Moon JH, Koo BK, Moon MK. Optimal high-density lipoprotein cholesterol cutoff for predicting cardiovas- cular disease: comparison of the Korean and US National Health and Nutrition Examination Surveys. J Clin Lipidol 2015;9:334-342.

24. Chandak GR, Ward KJ, Yajnik CS, et al. Triglyceride associated polymorphisms of the APOA5 gene have very different allele frequencies in Pune, India compared to Europeans. BMC Med Genet 2006;7:76.

25. Chhabra S, Narang R, Krishnan LR, et al. Apolipoprotein $\mathrm{C}_{3}$ SstI polymorphism and triglyceride levels in Asian Indians. BMC Genet 2002;3:9.

26. Hu EA, Pan A, Malik V, Sun Q. White rice consumption and risk of type 2 diabetes: meta-analysis and systematic review. BMJ 2012;344:e1454.

27. Lee MJ, Popkin BM, Kim S. The unique aspects of the nutrition transition in South Korea: the retention of healthful elements in their traditional diet. Public Health Nutr 2002;5:197-203.

28. Harbis A, Perdreau S, Vincent-Baudry S, et al. Glycemic and insulinemic meal responses modulate postprandial hepatic and intestinal lipoprotein accumulation in obese, insulin-resistant subjects. Am J Clin Nutr 2004;80:896902.

29. Mamo JC, James AP, Soares MJ, Griffiths DG, Purcell K, Schwenke JL. A low-protein diet exacerbates postprandial chylomicron concentration in moderately dyslipidaemic subjects in comparison to a lean red meat protein-enriched diet. Eur J Clin Nutr 2005;59:1142-1148.

30. Iso $H$, Naito $Y$, Sato $S$, et al. Serum triglycerides and risk of coronary heart disease among Japanese men and women. Am J Epidemiol 2001;153:490-499.

31. Acartürk E, Cayli M, Akpinar O, Attila G, Demir M. Relation between age and gender differences in plasma triglyceride concentrations and coronary artery disease in Southern Turkey. Clin Chim Acta 2004;339:123-128.

32. Hokanson JE, Austin MA. Plasma triglyceride level is a risk factor for cardiovascular disease independent of high-density lipoprotein cholesterol level: a meta-analysis of population-based prospective studies. J Cardiovasc Risk 1996;3:213-219.

33. Karim MN, Ahmed KR, Bukht MS, et al. Pattern and predictors of dyslipidemia in patients with type 2 diabetes mellitus. Diabetes Metab Syndr 2013;7:95-100.

34. Mero N, Malmstrom R, Steiner G, Taskinen MR, Syvanne M. Postprandial metabolism of apolipoprotein B-48- and B-100-containing particles in type 2 diabetes mellitus: relations to angiographically verified severity of coronary 
artery disease. Atherosclerosis 2000;150:167-177.

35. Syvanne M, Hilden H, Taskinen MR. Abnormal metabolism of postprandial lipoproteins in patients with non-insulin-dependent diabetes mellitus is not related to coronary artery disease. J Lipid Res 1994;35:15-26.

36. Haffner SM. Lipoprotein disorders associated with type 2 diabetes mellitus and insulin resistance. Am J Cardiol 2002;90:55i-61i. 